\title{
Surface Mechanical Properties of Comet 67P
}

\author{
W. Arnold ${ }^{1, \text { a }}$, H.-H. Fischer ${ }^{2}$, M. Knapmeyer ${ }^{3}$, and H. Krüger ${ }^{4}$, \\ ${ }^{1}$ Saarland University, Department of Materials Science and Engineering, Saarbrücken, Germany \\ ${ }^{2}$ DLR Microgravity User Support Centre, Cologne, Germany \\ ${ }^{3}$ DLR Institute of Planetary Research, Berlin, Germany \\ ${ }^{4}$ Max Planck Institute for Solar System Research, Göttingen, Germany
}

In August 2014 the ESA spacecraft Rosetta encountered the comet 67P/ChuryumovGerasimenko. The overall objective of the Rosetta mission was to determine physical and chemical properties of comet 67P. The orbiter of Rosetta was itself an instrument platform. It also carried the lander Philae that landed on the comet $\hat{Q}$ nucleus on November $12^{\text {th }} 2014$. Philae had ten different instruments on board including the Surface Electric Sounding and Acoustic Monitoring Experiment (SESAME) comprising the Comet Acoustic Surface Sounding Experiment (CASSE), the Dust Impact Monitor (DIM), and the electric impedance probe (PP). The mission ended on September $30^{\text {th }} 2016$ with the de-orbiting of Rosetta onto comet 67P.

CASSE sensors consisted of piezo-electric transmitters and accelerometers as receivers. They were mounted in the soles of Philaêิ landing-gear feet. The original operation principle of CASSE was to insonify the comet at frequencies from $0.5 \mathrm{kHz}$ to $1 \mathrm{kHz}$. CASSE was also used to measure the accelerations of the landing shock and the soleâ contact-resonances when the feet touched ground the first time on comet 67P at site Agilkia. The strength of the landing shock or the force acting between Philaeâ landing gear and the comet soil is determined on the one hand by the compliance of the material encountered at the landing location and on the other hand by the compliance of that part of the landing gear which touches the surface. Besides the absolute value of the force of the landing shock, the forced resonances of the soles of the feet can be used to obtain information on the local elastic modulus $\mathrm{E}$ and the compression strength $\stackrel{\mathrm{u}}{\mathrm{c}}_{\mathrm{c}}$ of the comet soil.

The acceleration signals at the first landing site Agilkia which occurred in the first seconds of the touchdown at an impact velocity of approx. $1 \mathrm{~m} / \mathrm{s}$ were recorded by CASSE. The inversion of the data yielded the compression strength and the elastic modulus of the cometary soil. Both are very low compared to commonly used engineering materials. The data support the concept that the elastic (E) and strength properties (U⿱⺈) of the comet material correspond to very porous solids constituted by the

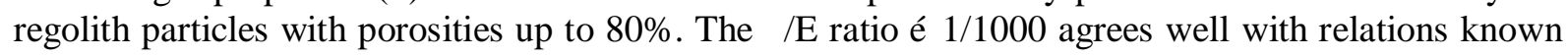
in material science.

On the comet surface at the final landing site Abydos, CASSE operated jointly with the MUPUS (Multi-purpose Sensors for Surface and Sub-Surface Science) instrument. The main goal of MUPUS was to measure the thermal properties of the surface material. To this end an instrumented rod was hammered into the ground. In addition, the hammering with stroke energies up to 4 Joule generated surface waves in the $100 \mathrm{~Hz}$ to $2 \mathrm{kHz}$ range which were detected by CASSE. Group arrival time differences between the three feet of Philae were measured and the Rayleigh wave velocity was derived. From the signals dispersion, one can conclude that comet 67P at Abydos is structurally layered.

The Dust Impact Monitor $\mathbf{Q}$ (DIM) PZT sensor elements aimed to detect and to derive the elasticplastic properties and the flux of the millimeter-sized dust-particle population that moves near the surface of the comet nucleus. During the descent of Philae, a signal was recorded of a dust particle by a sensor element and its diameter and elastic modulus were derived. A detailed analysis implied a porous particle whose properties lied close to aerogel particles having a porosity of å $90 \%$. Because their Youngố modulus is å $10 \mathrm{MPa}$, it was conjectured that the particle detected possessed a similar value.

In this presentation, an overview on the course of the Rosetta mission is given and data recorded by CASSE and DIM are presented. The SESAME measurement techniques used on comet $67 \mathrm{P}$ are compared to those used in non-destructive materials characterization using ultrasonic measurement techniques to measure the thickness of thin films by SAW dispersion, and to exploit contactresonances in dynamic atomic force microscopy, and in mechanical impedance testing. Finally, our comet data are compared with strength estimates made by aid of the data from other Rosetta observations. 